\title{
Bile salts and liver disease in childhood
}

\author{
NORMAN B. JAVITT \\ M.D., Ph.D. \\ Gastroenterology, New York Hospital-Cornell University Medical College
}

\begin{abstract}
Summary
Recognition of neonatal liver disease has been heavily dependent on the occurrence of jaundice. In most instances the jaundice is related to specific disturbances in bilirubin transport and other tests of liver function are normal. In contrast, hepatitis and other liver diseases not specifically affecting bilirubin transport often go undetected unless jaundice occurs.

The development of practical methods for the estimation of bile acids in serum has permitted an evaluation of hepatic excretory function in neonates and children independent of bilirubin excretion. Since bile acid excretion by the liver each day greatly exceeds bilirubin excretion it was not surprising to find that elevations in serum bile acids occur regularly in anicteric hepatitis. Because the excretion of bile acids generates canalicular bile flow a reduction in the capacity to excrete bile acids intimates the presence of cholestasis. Early cholestasis is not associated with hyperbilirubinaemia but as cholestasis becomes more severe, jaundice occurs and the possibility of biliary atresia arises in neonates. Serum bile acid patterns in neonates being evaluated for biliary atresia indicate two distinctive patterns. Those infants with severe cholestasis and patent bile ducts usually have predominantly cholic acid in serum. This observation is consistent with the bile acid patterns found in intrahepatic and extrahepatic cholestasis occurring in adult life. Infants found to have extrahepatic biliary atresia have marked elevations in the proportion of chenodeoxycholate in serum. Since elevations of chenodeoxycholate in serum are associated with hepatitis, the findings are consistent with the view that extrahepatic atresia is a rare sequalae of hepatitis.

Studies of bile acid metabolism and excretion give promise of providing further insight on the pathogenesis of cholestatic liver disease.
\end{abstract}

My interest in the metabolism and excretion of bile salts is an outgrowth of an interest in the excretory function of the liver (Anderson and Javitt, 1974). Both the liver and the kidney have metabolic functions and excretory functions. Usually the kidney is viewed almost exclusively as an excretory organ and the liver as a metabolic organ. However, the kidney metabolically is a small liver, even to the extent of $\overrightarrow{\vec{\omega}}$ glucose synthesis, or BSP conjugation or hydroxyla- $\frac{\rho}{0}$ tion of vitamin D. Similarly, the liver has an im- $\frac{0}{3}$ portant excretory function and actually bile contains small amounts of all the compounds that are ultra- $O$ filterable at the glomerulus.

In addition, the liver excretes a number of compounds into bile in high concentration, that normally of appear in only trace amounts in urine.

Figure 1 shows the organic anions present in bile $\frac{\text { ㅇ }}{}$ and the amount excreted over a $24 \mathrm{hr}$ period. Note $\vec{c}$ that bilirubin is a true pigment in that the relatively small amount in bile is responsible for its striking $\overparen{\Phi}$ colour. Actually it is the colourless bile salts that $\overrightarrow{0}$ represent the major organic anions in bile. The difference between bilirubin and bile salt as am excretory load on the liver becomes even more striking when one considers the enterohepatic circulation.

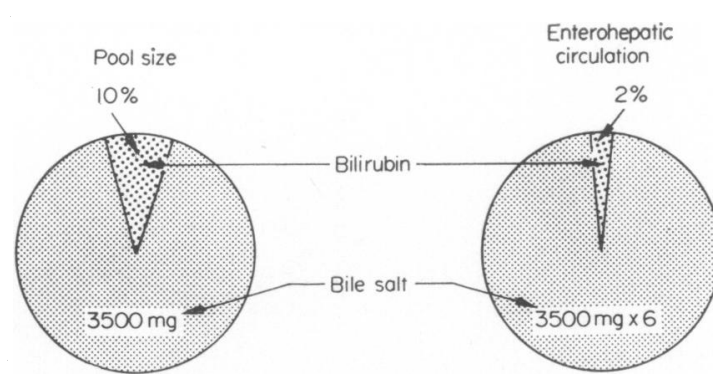

Fig. 1. Pool size and enterohepatic circulation of bile salt and bilirubin. Less bilirubin than bile salt is reabsorbed from the intestine and therefore bilirubin becomes an even smaller proportion of the total anion load.

Most of the bile salt entering the intestine is $N$ reabsorbed whereas most of the bilirubin is excreted. N No data are available on what fraction of the bili- 0 rubin might be reabsorbed from the intestine in a physiological setting, but assuming that a fourth $\frac{0}{\mathbb{D}}$ or $25 \%$ of the bilirubin is reabsorbed and reexcreted $\stackrel{5}{?}$ in bile this is considerably less than the $90 \%$ or more 0 reabsorption of bile acids from the intestine. Therefore, in terms of the daily excretory load on the liver, the bile salts greatly exceed bilirubin. 
From a quantitative point of view, therefore, one would guess that disturbances in hepatic excretory function are more likely to be detected by changes in bile acid excretion (Kaplowitz, Kok and Javitt, 1973) rather than changes in bilirubin excretion. However, it is only recently that we have had the technical capability of estimating changes in bile acid excretion and therefore practically all our concepts of hepatic excretion have been based on bilirubin metabolism. The situation is changing rapidly and it is already apparent that estimation of serum bile acid levels is a far more sensitive way of evaluating hepatic excretory function than the estimation of serum bilirubin levels. In particular a lot of liver disease in infancy and childhood is anicteric, and better methods must be found for early recognition of the problem.

Figure 2 illustrates this point. Dr Saul Krugman was kind enough to give us thirty-six sera of patients exposed to hepatitis B virus. All of the samples were $0.2 \mathrm{ml}$ which is the smallest volume ever analysed for bile acids. We used our published method (Ali and Javitt, 1970) by just proportionally reducing all the materials. Sample size is therefore really no longer any reason for not obtaining more information in neonates. When we sent Dr Krugman our results he compared them to the SGOT levels in serum and the antigenaemia. Although the anti-

Patient

Se $\begin{aligned} & \overline{X X X X X X X X X X X X X X} \\ & 000000000000000000\end{aligned}$

Br $\begin{aligned} & X X X X X X X X X X X X X X X X \\ & 0000000000000000000000\end{aligned}$

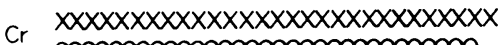

00000000000000000000000000000

Sk $X X X X X X X X X X X X X X X X X X X X X X X X X X X X X X X X X$ 0000000000000000000000000000000000000000

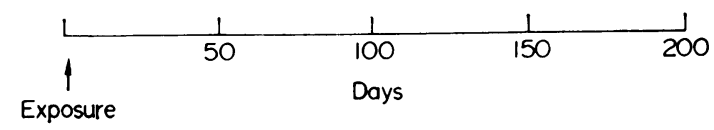

FIg. 2. Serum abnormalities following exposure to hepatitis B virus. Serum bile acid levels were analysed in four individuals exposed to hepatitis B virus. In each instance an abnormal elevation occurred usually following the elevation in SGOT. - - antigenaemia; XXX, GOuT; OOOO, bile acid. Symbols extend to first day test became abnormal. genaemia always occurred earlier, an elevation in serum bile acid occurred in all the patients close to the time of transaminase increase. Needless to say, none of the sera had elevations in bilirubin. This is probably the first evidence that disturbances in hepatic excretory function occur regularly in anicteric hepatitis.

The neonatologist has a special interest in bilirubin, perhaps more in its conjugation than its excretion. The problem of Kernicterus in the neonate is most serious, and fortunately good preventive techniques have been developed. The neonatologist may not know what the bile acid concentrations are in the sera of these infants, and, parenthetically, whether they contribute in any way to the diffusibility of bilirubin into the central nervous system. However, we assume, perhaps incorrectly, that serum bile acid level is always normal and that the only abnormality is in bilirubin metabolism. This indeed, is a definition of hyperbilirubinaemia, an abnormality exclusively in bilirubin metabolism. Except in the newborn period, hyperbilirubinaemia per se, is a benign disorder quite compatible with a long and colourful life. (Except perhaps for the very rare problem of the Crigler-Najjar syndrome.)

It would be nice if we knew as much about bile acid metabolism in the newborn as we know about bilirubin metabolism, but there is very little information available. There follows an outline of the present concepts.

Figure 3 illustrates the studies of Back and Ross (1973). It shows the proportions of mono-, di- and trihydroxy bile acids in normal human meconium obtained from thirteen premature neonates and eleven full term neonates. The origin of all the bile

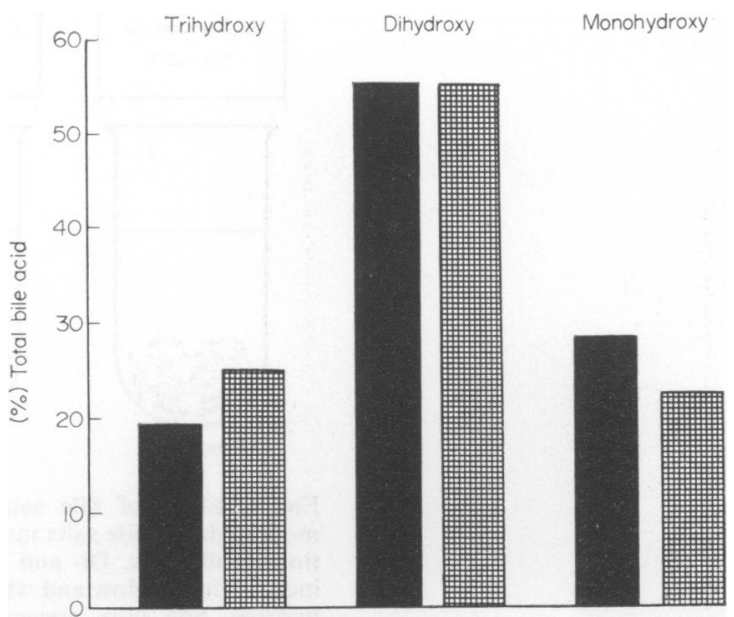

Fig. 3. Proportion of mono-, di- and trihydroxy bile acids in human meconium. (See Back and Ross, 1973.) Solid, premature; hatched, full term. 
acids is not known. Some of them could have been transported across the placenta and others made in the foetal liver. In either event most of the bile acids found were considered to be synthesized in the liver and not the result of intestinal bacterial activity.

If this premise is correct it becomes very difficult to explain the presence of monohydroxy bile acids according to the currently proposed theory of bile acid synthesis (Danielsson and Tchen, 1968).

The metabolic pathway for the synthesis of bile acids from cholesterol is as follows. The cholesterol molecule can be divided into two parts, a steroid ring and a hydrocarbon side chain. The current belief is that in the adult, the metabolism of cholesterol to bile acid begins with the addition of a hydroxy group at the seven position to form $7 \alpha$-hydroxycholesterol. Once this rate limiting step occurs, there is a very rapid transformation of the steroid moiety into either di- or trihydroxycoprostane and then oxidation of $\frac{\$}{\Phi}$ the side chain to a C-24 bile acid. The important $\cong$

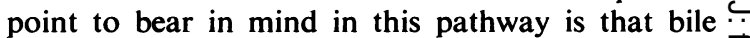
acids with only one hydroxyl group are not formed $\overrightarrow{\vec{\rho}}$ as intermediates. The end products are the dihydroxy bile acids, chenodeoxycholic acid and a trihydroxy bile acid, cholic acid.

Although this theory of bile acid synthesis may be entirely correct for the adult, it raises some questions in regard to foetal and neonatal life. In order to कs explain the in vivo production of monohydroxy bile $\overrightarrow{0}$ acids one has to postulate an alternate pathway. One $\overrightarrow{\vec{H}}$ possibility is that bile acid synthesis from cholesterol $\omega_{\mathscr{\rho}}$ begins with side chain oxidation of cholesterol to $\frac{}{0}$ 26-hydroxycholesterol. This alternate pathway has been investigated in our laboratory by a series of studies in both man and animal. Emerman (Emerman, Wachtel and Javitt, 1968) was able to isolate

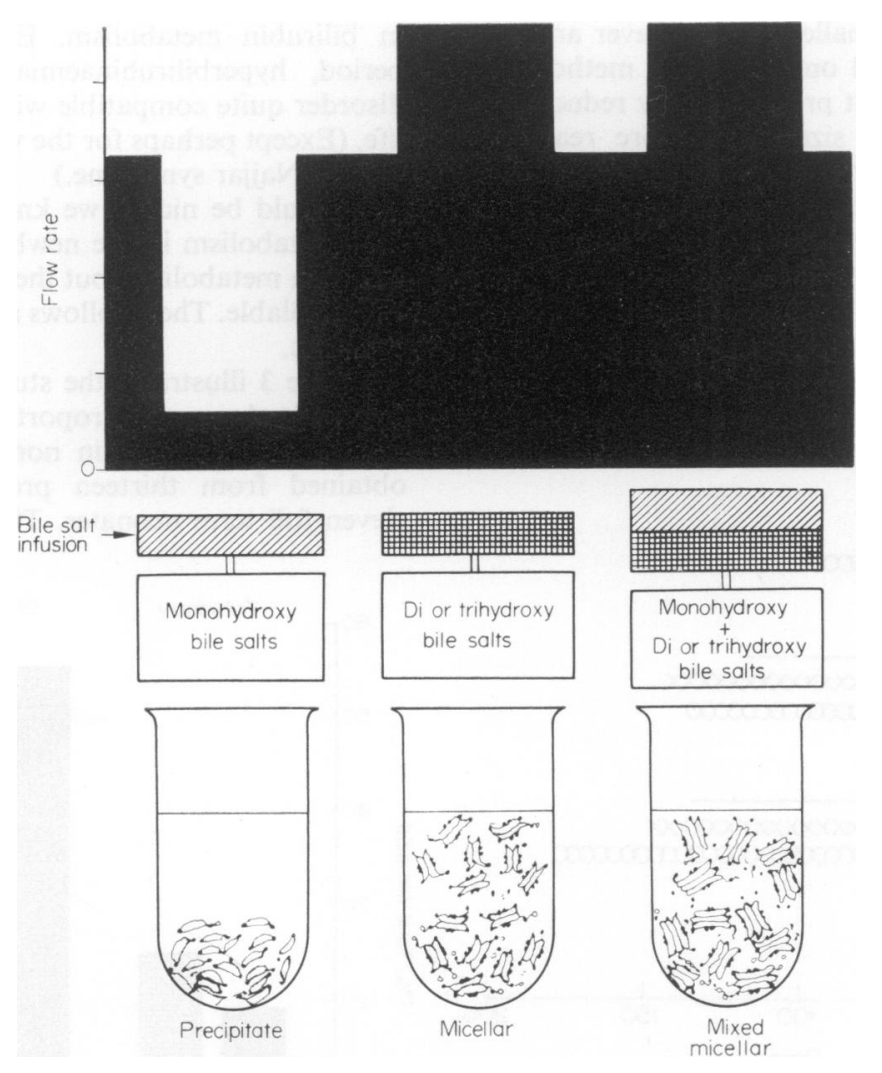

FIG. 4. Effect of bile salts on bile flow. Infusion of monohydroxy bile salts into rats causes a prompt reduction in bile flow. $\mathrm{Di}$ - and trihydroxy bile salts cause an increase in bile flow and when given together with monohydroxy bile salts prevent the cholestatic effect. The mechanism is believed to be related to precipitation of monohydroxy bile salts in the biliary tree and is prevented by micellar solubilization. 
monohydroxy bile acids from animals given 26hydroxycholesterol, chiefly, the one found in the urine and meconium of infants. Dr Karl Anderson has shown that in man both $7 \alpha$, and 26-hydroxycholesterol are metabolized to chenodeoxycholic and cholic acids (Anderson, Kok and Javitt, 1972). More recently, Dr Uri Lavy (Lavy, Burstein and Javitt, 1973) has found that normal human meconium contains relatively large amounts of 26-hydroxycholesterol but no $7 \alpha$-hydroxycholesterol. Thus we believe that both pathways may exist in man.

Our interest in the metabolic pathways for bile acid synthesis came about when we found that monohydroxy bile acids had a special effect on bile flow (Javitt and Emerman, 1968). They completely suppress flow when given in large amounts. This phenomenon is illustrated in Fig. 4.

If one infuses a monohydroxy bile acid into a rat or hamster, bile flow immediately falls and a cholestatic syndrome ensues and lasts from 24 to $48 \mathrm{hr}$ followed by a complete recovery. Di- and trihydroxy bile acids always increase bile flow and will prevent the cholestasis caused by monohydroxy bile acids by forming soluble complexes with them.

With all this work behind us, we began to investigate the cholestatic syndromes occurring in the newborn period, thinking that some of this very exciting experimental work must prove to be the pathophysiological mechanism underlying neonatal cholestatic liver disease. We have made some interesting observations, perhaps more important than we were planning. However, we have not as yet established a metabolic error in bile acid synthesis as a cause of a neonatal cholestatic syndrome.

Let us review our studies in infants which have been confined almost exclusively to an analysis of serum bile acid patterns.

Since the normal liver has a great capacity to remove bile acids from the blood, the normal level in serum is about $1-2 \mu \mathrm{g} / \mathrm{ml}$. At this very low level we can not be as certain of the identification of each bile acid in serum. However, in various liver diseases the concentration rises from 10 to 200 fold and at these levels we can be quite certain about the proportion of each bile acid in serum although we are less certain how accurately the proportions in serum reflect the proportions in the gallbladder or the intestines.

In considering serum bile acid patterns in diseases of the liver and biliary tract one can evaluate both the proportion of each bile acid in serum and the total concentration in serum.

Figure 5 summarizes our experience in evaluating adult human liver disease. If the ratio of chenodeoxycholate to cholate is less than 0.4 we can be certain we are dealing with hepatitis or cirrhosis. If the ratio is greater than 4 we can be quite certain we are dealing with a cholestatic syndrome. By a cholestatic syndrome we mean that there is a disturbance in bile flow with little or no evidence of cell necrosis. The disturbance may be related to mechanical obstruction or some metabolic failure in the flow generating system of the liver cell. In hepatitis and cirrhosis the total levels are less than obstructive disease. Clearly there is a large group in which the ratio and the totals do not give us a great deal of confidence in the diagnosis.

Now when we began to evaluate serum bile acid patterns in the newborn period (Javitt et al., 1973), we accidentally came across some very striking patterns that puzzled us greatly.

Figure 6 shows a serum bile acid pattern of an infant that is being followed at New York University Medical Center and was our first experience. We use an internal standard that has a retention time longer than any of the normal bile acids and therefore it is unlikely that we would miss one in our analysis. Note that the serum contained more than $90 \%$ chenodeoxycholate with a rather high total of 133 $\mu \mathrm{g} / \mathrm{ml}$. The child was about 3 months old and had been explored and found to have extrahepatic biliary atresia. A pattern like this does not exist in an adult. The only pattern that comes close to it, is that seen in advanced, pre-terminal cirrhosis, where one sees almost all chenodeoxycholate but the total is much lower. The lower total in cirrhosis is presumably related to the very low synthesis of bile acids.

Naturally, we began to analyse further specimens from around the country and rather quickly and most surprisingly it became apparent to us that infants

\begin{tabular}{lccc}
\hline & $\begin{array}{c}\text { Hepatitis } \\
\text { cirrhosis }\end{array}$ & Indeterminate & $\begin{array}{c}\text { Cholestasis } \\
\text { intra or extra } \\
\text { hepatic }\end{array}$ \\
\hline $\begin{array}{l}\text { Ratio } \\
\text { Cholate/chenodeoxycholate }\end{array}$ & $<0.4$ & $0.4-4$ & $>4$ \\
$\begin{array}{c}\text { Total } \\
(\mu \mathrm{g} / \mathrm{ml})\end{array}$ & $<40$ & $40-100$ & $>100$ \\
\hline
\end{tabular}

FIg. 5. Serum bile acid patterns in adult liver disease. Bile acid patterns can occasionally be diagnostically helpful, but often there are values that are indeterminate. 


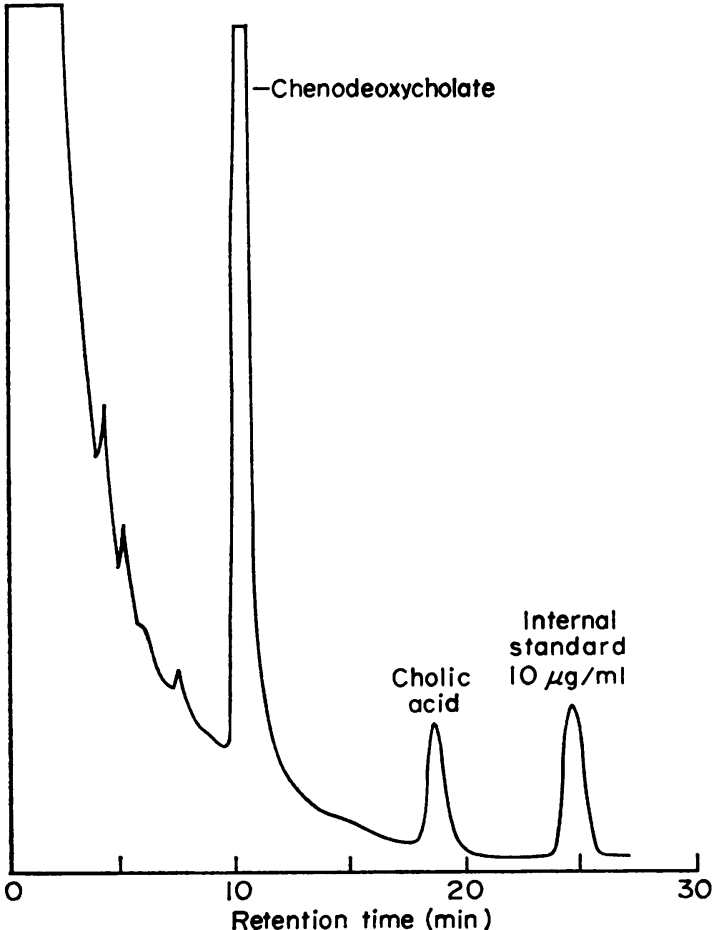

FIG. 6. Quantitative analysis of serum bile acid pattern by gas-liquid chromatography. The total value was 133 $\mu \mathrm{g} / \mathrm{ml}$ (normal $1-2 \mu \mathrm{g} / \mathrm{ml}$ ). This patient was found to have extrahepatic biliary atresia.

diagnosed as extrahepatic atresia had patterns with a strikingly high proportion of chenodeoxycholate.

One reason this was a surprise to us is that it had not been commented on previously. Sandburg (1970) reported five patients with extrahepatic atresia only two of whom had a preponderance of chenodeoxycholate. We have tried to obtain more information about these patients but it is no longer available. The important point is when in relation to the onset of the disease were the samples analysed? Since these infants may live for several years the pattern could vary considerably. Perhaps they would initially show a predominance of chenodeoxycholate, then change to a cholate pattern as the hepatitis subsides and then finally as cirrhosis develops, become predominantly chenodeoxycholate again.

Since our initial observation we have had the opportunity to analyse serum bile acid patterns in $\stackrel{?}{?}$ twenty-five infants and children sent to us from allo over the U.S. and the results are summarized in Fig. 흘 7. The surprise is that extrahepatic atresia is segre- $\frac{\bar{\sigma}}{\bar{D}}$ gating with hepatitis and not with other cholestatic $\stackrel{\varnothing}{\varrho}$ syndromes. We think that our data strongly suggest the view that some paediatricians have had for $a_{\vec{A}}$ long time. Extrahepatic atresia may be, in most $?$ instances, an acquired sequelae of neonatal hepatitis $\vec{\omega}$ and not a primary foetal abnormality in the anatomic $\stackrel{\omega}{\circ}$ formation of the biliary tree.

If we accept as a working hypothesis that at least 3 . some types of extrahepatic biliary atresia are a con- $y$ sequence of hepatitis, how does this affect our $\because$ approach to the problem? It is certain that one approach must be a co-operative one. It is a rare disease with a variable course and unless physicians $\rightarrow$ develop together some uniform therapeutic approach음 we will never know which treatment may be most successful. This does not refer to a uniform surgical $\_$

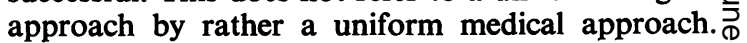
Many of these neonates really have anicteric hepatitis $\overrightarrow{0}$ and the first sign of jaundice is evidence of partig atresia. At first only a portion of the duct system my become occluded and if the inflammation subsidesso the remaining patent system compensates and thes jaundice disappears. In others, the closing of the $\bar{\partial}$ ducts follows a relentlessly progressive course. If $\stackrel{0}{\not}$ this hypothesis is correct, then we need some way of $\stackrel{\mathbb{Q}}{2}$ detecting anicteric hepatitis early, perhaps routine $\overrightarrow{\vec{O}}$ immunoassay for serum bile acids. There are other 3 aspects of our studies.

There are a group of neonates who develop? jaundice as part of a cholestatic syndrome early ino life and often the hyperbilirubinaemia will fall spontaneously to levels of 3-4 mg/\%. Although they have patent bile ducts, they are often explored and. it is common practice to do some type of venting procedure usually a choledochoenterostomy. Many of these infants will respond to either phenobarbitalo or cholestyramine and surgery should not be done $>$ until a diagnostic or therapeutic trial of these medications is carried out (Morrissey and Javitt, 1973). N

\begin{tabular}{lccc}
\hline & $\begin{array}{c}\text { Hepatitis } \\
\text { extrahepatic } \\
\text { atresia }\end{array}$ & Indeterminate & $\begin{array}{c}\text { Cholestasis } \\
\text { patent bile ducts }\end{array}$ \\
\hline $\begin{array}{l}\text { Ratio } \\
\text { Cholate/chenodeoxycholate }\end{array}$ & $<1$ & $?$ & $>1$ \\
$\begin{array}{l}\text { Total } \\
(\mu \mathrm{g} / \mathrm{ml})\end{array}$ & $30-130$ & $?$ & $100-400$ \\
\hline
\end{tabular}

Fig. 7. Serum bile acid patterns in neonatal liver disease. Neonates with hepatitis and extrahepatic biliary atresia segregate together. 


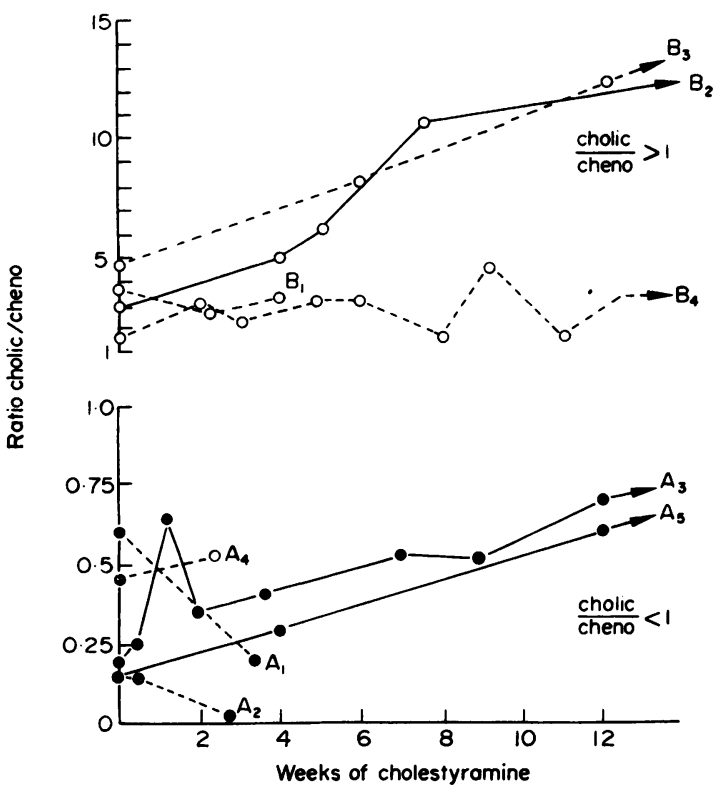

FIG. 8. Effect of cholestyramine feeding in infants (solid line) and children (interrupted line) with patent bile ducts and with extrahepatic biliary atresia. Cholestatic syndromes with patent bile ducts tend to have cholate/ chenodeoxycholate ratios greater than one and rise progressively on cholestyramine feeding (from L. Schiff, Diseases of the Liver, fourth Ed.) (in press.) -0-, patent bile ducts; - - extrahepatic biliary atresia.

Figure 8 summarizes our early studies with cholestyramine (Javitt et al., 1973).

Infants with patent bile ducts have a cholate to chenodeoxycholate ratio usually greater than one, and the ratio increases as the total concentration falls. The change in ratio as shown here is the most characteristic feature of the cholestyramine sequence and establishes the patency of the bile ducts. It occurs because chenodeoxycholate is bound to the resin in greater proportion than cholate. It is very difficult to believe that children who respond to phenobarbital or cholestyramine and lose their jaundice really have a primary anatomic defect. Rather they may represent defects in bile formation. It is not known what these abnormalities are. There may be a variety of abnormalities.

Figure 9 is an attempt to classify the types of defects that may be expressed clinically as a cholestatic syndrome. The definition of a cholestatic syndrome as a reduction in canalicular bile flow is operationally useful (Javitt, 1971).

Operationally we separate the flow generating mechanisms in the canaliculus into those related or dependent on bile salt excretion and those independent of it. Phenobarbital is known to increase canalicular bile flow (Berthelot et al., 1970) independent of

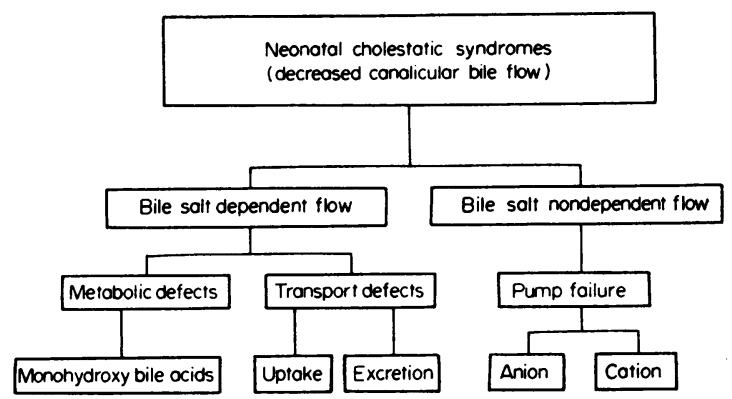

FIG. 9. Neonatal cholestatic syndromes; cholestatic syndromes are considered to be disturbances in the canalicular flow generating mechanism of the liver.

any effect it may have on total bile acid excretion. Other agents, such as ethacrynic acid (Shaw et al., 1972) and ouabain (Graf, Korn and Peterlik, 1972), also increase canalicular bile flow without apparently affecting bile salt excretion. These observations imply the existence of ionic pumps that may be governed by mechanisms independent of bile salt concentration. Instances of neonatal cholestasis have been reported in which the patients respond to phenobarbital and not cholestyramine (Ballow et al., 1973) and perhaps these infants represent examples of defects in bile salt nondependent flow.

Other instances occur in which cholestyramine appears to have a much more beneficial effect than phenobarbital (Sharp and Mirkin, 1972). Since the only established effect of cholestyramine is its capacity to bind bile acids it implies that it may be correcting some aspect of bile salt transport and is therefore related to bile salt dependent flow.

Although monohydroxy bile acids have been found as the conjugated and sulphated derivative in the urine of infants with cholestatic syndromes, we have no precise idea of whether they initiate or perpetuate the cholestasis. Preliminary studies in animals (Javitt, 1973) indicates that the conjugated and sulphated compound do not affect bile flow but the situation could be very different in man.

\section{References}

AlI, S.S. \& JAVITT, N.B. (1970) Quantitative estimation of bile salts in serum. Canadian Journal of Biochemistry, 48, 1054.

ANDERSON, K. \& JAVITT, N.B. (1974) Bile in the LiverMolecular Biology of Function and Malfunction (Ed. by F. F. Becker). Marcel Dekker, Inc., New York and London (in press).

ANDERSON, K.E., KoK, E. \& JavitT, N.B. (1972) Bile acid synthesis in man: Metabolism of $7 \alpha$-hydroxycholesterol${ }^{14} \mathrm{C}$ and 26-hydroxy-cholesterol- ${ }^{3} \mathrm{H}$. Journal of Clinical Investigation, 51, 112.

BACK, P. \& Ross, K. (1973) Identification of 3ß-hydroxy-5cholenoic acid in human meconium. Hoppe-Sayler's $Z$. Physiological Chemistry, 354, 83. 
Ballow, M., Margolis, C.Z., Schachtel, B. \& Hsia, V.E. (1973) Progressive familial intrahepatic cholestasis. Pediatrics, 51, 998.

Berthelot, P., Erlinger, S., Dhumeaux, D. \& Preaux, A-M. (1970) Mechanism of phenobarbital induced hypercholeresis in the rat. American Journal of Physiology, 219, 809.

Danielsson, H. \& Tchen, T.T. (1968) Steroid metabolism. In: Metabolic Pathways (Ed. by D. M. Greenberg). Volume II. Lipids, steroids and carotenoids. 3rd ed. Pp. 117-168. Academic Press, New York.

Graf, J., Korn, P. \& Peterlik, M. (1972) Choleretic effects of ouabain and ethacrynic acid in the isolated perfused rat liver. Naunyn-Schmed. Archives of Pharmacology, 272, 230.

JAVitT, N.B. \& EMERMAN, S. (1968) Effect of sodium taurolithocholate on bile flow and bile acid excretion. Journal of Clinical Investigation, 47, 1002.

JAVITT, N.B. (1973) Excretion of monohydroxy bile acid ester sulfates in the rat. In: The Liver. Quantitative Aspects of Structure and Function (Ed. by G. Paumgartner and R. Preisig). S. Karger, Basel.

JavitT, N.B. (1971) The cholestatic syndrome. American Journal of Medicine, 51, 637.

JavitT, N., Morrissey, K.P., Siegel, E., Goldberg, H., Gartner, L.M., Hollander, M. \& KoK, E. (1973)
Cholestatic syndromes in infancy. Diagnostic value of $\frac{\mathbb{Q}}{\overparen{Q}}$ serum bile acid patterns and cholestyramine administra- $\varrho$ tion. Pediatric Research, 7, 119.

KaPlowitz, N., KoK, E. \& JavitT, N.B. (1973) Post- $\Rightarrow$ prandial serum bile acid for the detection of hepatobiliary $\stackrel{\oplus}{\longrightarrow}$ disease. Journal of the American Medical Association, 225, 292.

LAVY, U., Burstein, S. \& JavitT, N.B. (1973) Fetal bile acid metabolism: Quantitation of 26-hydroxycholesterol and $7 \alpha$-hydroxy-cholesterol in human meconium. Gastroenterology, 65, 556.

MORRISSEY, K.P. \& JAVITT, N.B. (1973) Extrahepatic biliary atresia: Diagnosis by serum bile acid patterns and response to cholestyramine. Surgery, 74 (1), 116.

SANDBERG, D.H. (1970) Bile acid concentrations in serum during infancy and childhood. Pediatric Research, 4, 262.

ShARP, H.L. \& MiRKIN, B.L. (1972) Effect of phenobarbital $\frac{\partial}{\partial}$ on hyperbilirubinemia, bile acid metabolism and microsomal enzyme activity in chronic intrahepatic cholestasis of childhood. Journal of Pediatrics, 81, 116.

Shaw, H., CAPLe, I. \& Heath, T. (1972) Effect of ethacrynic acid on bile formation in sheep, dogs, rats, guinea-pig and rabbits. Journal of Pharmacology and Experimental Therapeutics, 182, 27.

WACHTEl, N., EMERMAN, S. \& JAVitT, N.B. (1968) Metabolism of cholest-5-ene-3ß, 26-diol in the rat and hamster. Journal of Biologic Chemistry, 243, 5207.

\section{Discussion}

Dr Murphy of Birmingham expressed concern about using phenobarbitone in the presence of a possible metabolic defect in bile salt metabolism. It seemed possible that this might aggravate the situation by stimulating pathways which could, for example, increase production of monohydroxy bile salts. Dr Murphy felt cholestyramine might be the more appropriate agent to use. Dr Javitt stated that they have not carried out any studies on the effect of phenobarbitone on bile acid metabolism and agreed that one had to be aware of such theoretical possibilities, but pointed out that there were some children in whom phenobarbitone did seem to work.
$\mathrm{He}$, too, much preferred cholestyramine since action on bile salts in the bowel is known and also if serum bile salts were altered from its use it would be possible to speculate as to how this has happened. He suggested that both agents might be used in sequence and all possible parameters in bile salt metabolism measured.

Dr Haas of Torquay commented that neonatal cholestasis often carries a good prognosis and the patients get better whatever is done. Dr Javitt agreed that this was so and that evaluation of the effects of treatment was thus difficult. Some children do have persisting symptoms and require treatment.

\section{General discussion on pathology and pathogenesis}

Professor Aagenaes of Oslo made a plea that the term 'Neonatal Hepatitis' or 'Neonatal Hepatitis Syndrome' be dropped from the nomenclature of neonatal liver disease since the word 'hepatitis' implies infection which appears to be a rare cause of neonatal obstructive jaundice. He suggested that a more neutral term such as 'Intrahepatic cholestasis' would be a more correct term for this clinical entity.

Dr Danks of Melbourne felt it was very difficult to get an all-embracing terminology. He used the term 'Neonatal Hepatitis' for children in whom liver biopsy showed a degree of cholestasis with liver cell necrosis and some reaction to these two abnormal ties in the form of inflammatory cell infiltrate, fibroblastic proliferation and the development of multinucleated cells. This definition would exclude patients with mild disease who did not have a liver biopsy and would include such well defined genetic 0 entities as galactosaemia or hereditary fructose $\bar{\Phi}$ intolerance; conditions which could not be dis- ? tinguished on histological grounds. He thus found it simplest to use the term 'Neonatal Hepatitis' to cover everything, including those with predominant liver cell necrosis or predominant cholestasis, and 